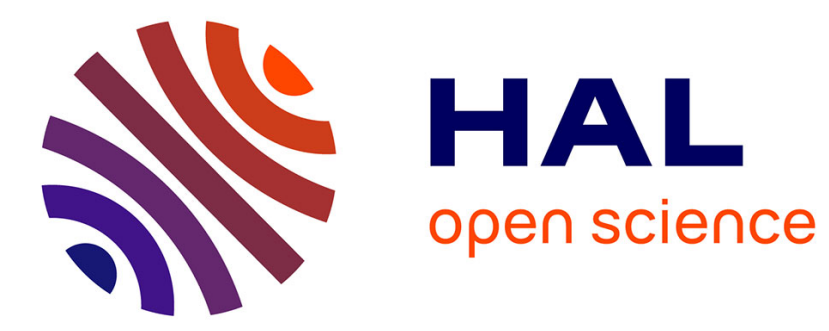

\title{
La Roumanie, terre d'émigration et de dépopulation
}

Gérard-François Dumont, Régis Flamand

\section{To cite this version:}

Gérard-François Dumont, Régis Flamand. La Roumanie, terre d'émigration et de dépopulation. Population et avenir, 2006, 671, pp.15-17. 10.3917/popav.680.0015 . halshs-00770363

\section{HAL Id: halshs-00770363 \\ https://shs.hal.science/halshs-00770363}

Submitted on 15 Nov 2013

HAL is a multi-disciplinary open access archive for the deposit and dissemination of scientific research documents, whether they are published or not. The documents may come from teaching and research institutions in France or abroad, or from public or private research centers.
L'archive ouverte pluridisciplinaire HAL, est destinée au dépôt et à la diffusion de documents scientifiques de niveau recherche, publiés ou non, émanant des établissements d'enseignement et de recherche français ou étrangers, des laboratoires publics ou privés. 


\section{La Roumanie, terre d'émigration et de dépopulation}

Le sixième élargissement ${ }^{1}$ de l'Union européenne concerne la Roumanie et la Bulgarie $^{2}$. Dans ces deux pays, avec une évolution géodémographique résultant de l'héritage du régime communiste, la baisse de la population est le trait majeur des années 2000. Examinons d'abord les particularités de la répartition spatiale de la population roumaine, puis les évolutions en cours.
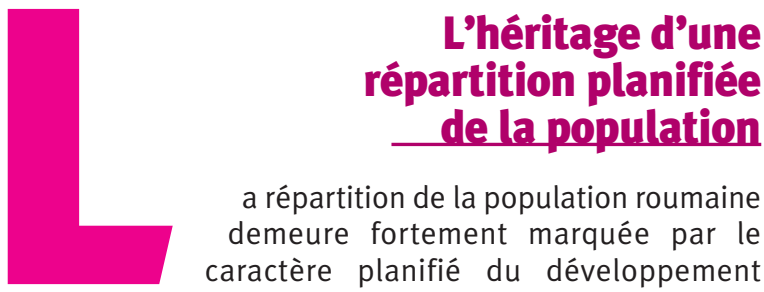

a répartition de la population roumaine demeure fortement marquée par le caractère planifié du développement socio-économique du pays de la période de l'après-guerre. Le processus d'industrialisation étant alors considéré comme la clé du développement, l'industrie lourde est privilégiée avec un déplacement des populations des campagnes. La réforme administrative du territoire de 1968, remplaçant une structure en grandes régions par un maillage de plus petites unités, les départements, susceptibles de stimuler la croissance de villes moyennes et ainsi de diffuser plus efficacement le développement, est un autre facteur de changement. Ainsi dans la période 1966-1977, les nouveaux pôles affichent des taux plus élevés que les anciennes capitales régionales, certaines villes étant bénéficiaires de l'encouragement unilatéral à l'industrie, alors que le secteur des services est pratiquement laissé à l'abandon.

\section{L'armature urbaine}

Ainsi, entre les recensements de 1977 , en plein règne d'un système économique à planification centralisée, et celui de 1992, soit au commencement de la transition vers l'économie de marché, la population roumaine connaît d'importantes redistributions géographiques ${ }^{3}$. Entre les villes chefs-lieux de départements (en général la ville la plus peuplée) et les villes qui suivent, les différences de poids démographique sont importantes, résultat du centralisme encouragé, sinon imposé, par l'ancien régime.

Après l'effondrement du système communiste, les grandes villes enregistrent une forte croissance démographique due à la levée des limitations des migrations

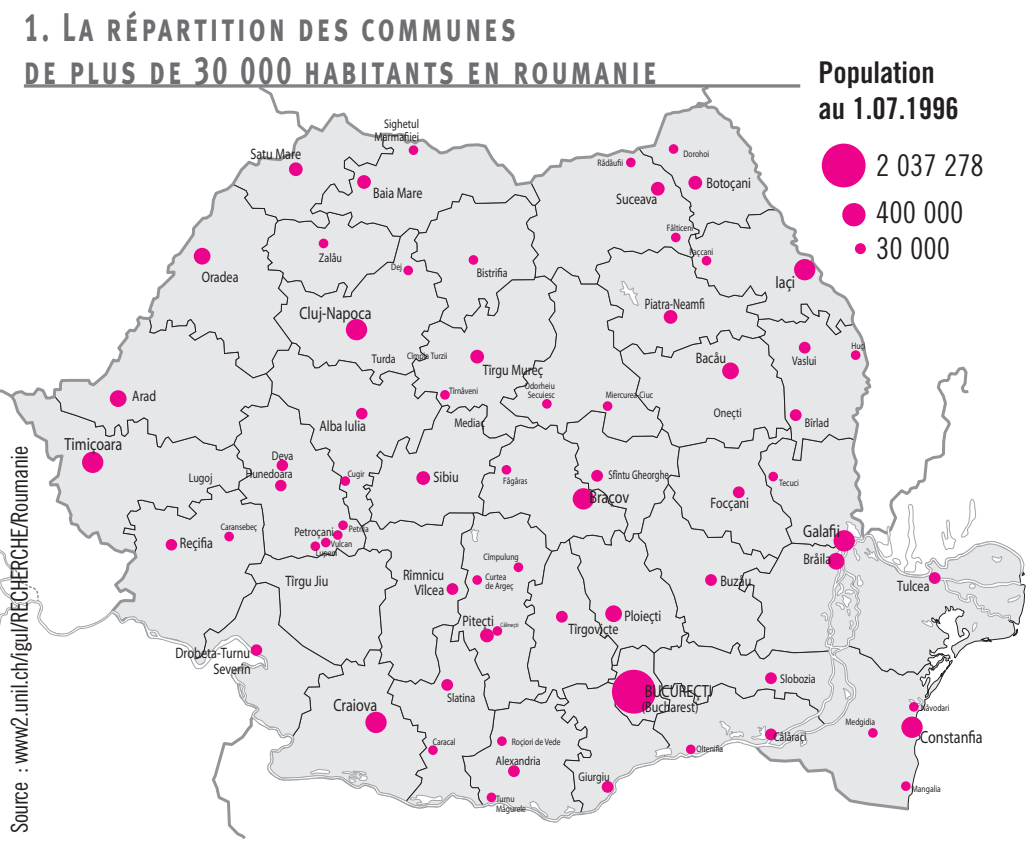

intérieures, à la réduction du nombre des migrations ponctuelles liées au travail et, partiellement, à la tentative de revitaliser les relations au bénéfice des villes anciennes auparavant délaissées ${ }^{4}$.

Dans les années 2000, du fait d'une natalité très basse, d'une forte émigration internationale et du départ de minorités (notamment allemande), la population de 166 agglomérations (sur 204) est en baisse, souvent dans des proportions importantes. Parallèlement, 8 agglomérations n'atteignent plus les 10 ooo habitants, tandis qu'une seule a franchi ce seuil dans l'autre sens.

L'agglomération de Bucarest (1,9 million d'habitants) est six fois plus peuplée que la deuxième agglomération du pays. Concernant les « grandes villes secondaires », 7 agglomérations se tiennent entre 328000 et 303000 habitants, de sorte qu'il n'y a pas « une » deuxième agglomération de Roumanie, mais potentiellement " 7 deuxièmes agglomérations » qui occupent cette place changeante au gré des recensements. 25 agglomérations dépassent les 100000 habitants.

\section{La dépopulation 5 roumaine}

La Roumanie totalise 21,6 millions d'habitants en 2006 sur $239000 \mathrm{~km}^{2}$, soit une densité moyenne de population de 90 habitants $/ \mathrm{km}^{26}$. À la fin du communisme, dans un premier temps, en 1990-1991, la décroissance de la population est exclusivement due à l'émigration, car le solde migratoire négatif est supérieur à
Gérard-François

Dumont

et Régis Flamand

1. Au $1^{\text {er }}$ janvier 2007 Sur les effets du cinquième, cf. "L'élargissement démographique de I'Union européenne », Population \& Avenir, $n^{\circ} 661$, janv.-févr. 2003. 2. Cf. Dumont GérardFrancois., Sougareva, "La Bulgarie en crise démographique », Population \& Avenir, $\mathrm{n}^{\circ}$ 671, janvier février 2005.

3. Le 3 mars 1978 , le gouvernement roumain décide de détruire 7000 villages sur les 13000 que compte le pays (5000 le seront) et déplace les populations rurales vers 600 « agro villages » sous le prétexte officiel de gagner 300000 hectares de terres cultivables pour l'agriculture. Mais il s'agit de mieux contrôler les populations rurales en les déracinant. 
l'accroissement naturel encore positif. Puis, depuis 1992, la Roumanie additionne chaque année un solde migratoire négatif, et une décroissance naturelle, les décès étant supérieur aux naissances.

Entre 2006 et 2025, donc en 20 ans, selon la projection moyenne, la population diminuerait à nouveau, d'environ 3,5 millions d'habitants. Dans le même temps, le vieillissement de la population s'accélèrerait, tandis que, depuis 2005 , la population active diminue. La situation de dépopulation des années 2000 se situe dans une évolution heurtée de la natalité depuis plus d'un demi-siècle.

\section{LA POPULATION EN ROUMANIE}

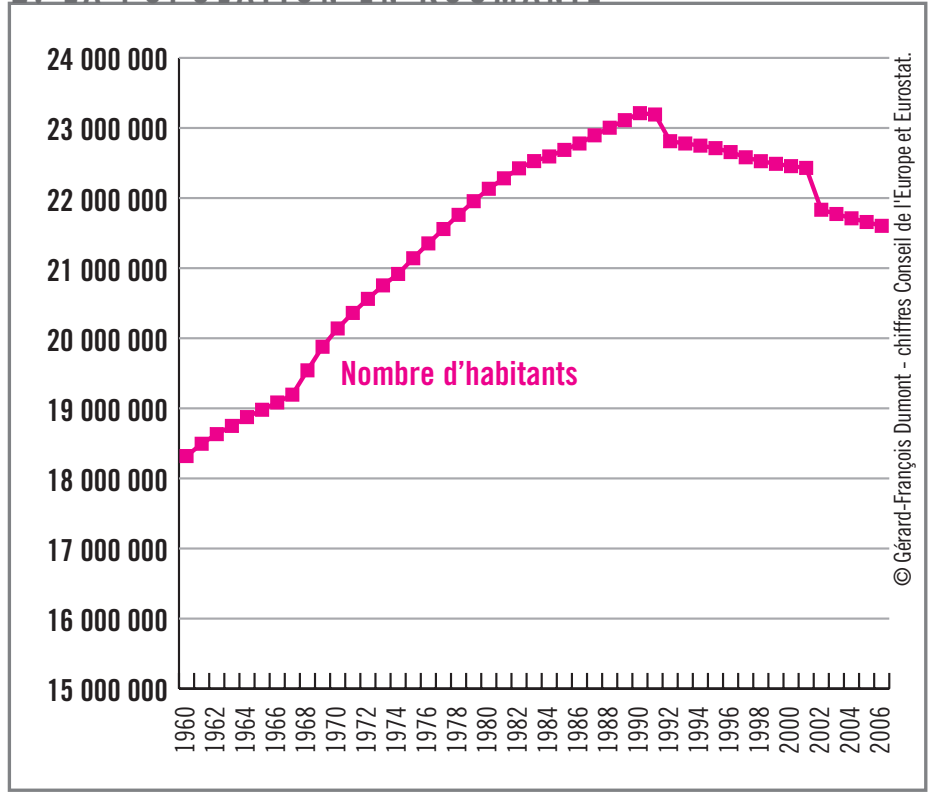

\section{LE MOUVEMENT NATURELEN ROUMANIE}

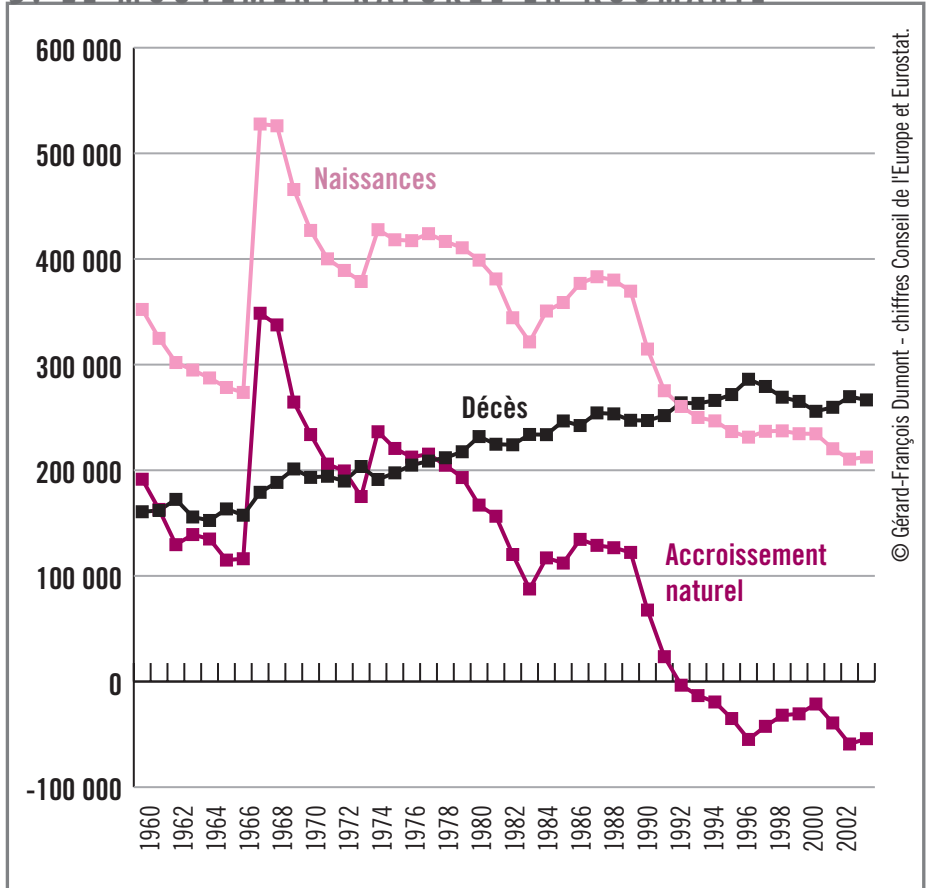

\section{La chute de la natalité}

Au lendemain de la Seconde Guerre mondiale, en Roumanie comme dans les autres pays communistes européens ${ }^{7}$, les comportements démographiques se modifient. Après 1956, date de la libéralisation de l'avortement, la natalité diminue fortement. En 1965, la Roumanie est en avant-dernière position en Europe pour le taux de natalité, devant la Hongrie. Ce résultat s'explique par le fait que les salaires demeurent bas, mais suffisants à condition de ne pas avoir d'enfants, ou un seul au maximum, même si le gouvernement apporte un soutien financier pour les familles nombreuses (soutien loin de compenser les coûts engendrés). Il peut s'expliquer aussi par l'entrée massive des femmes sur le marché du travail, et par la crise du logement.

Alors, comme les autres pays communistes, le gouvernement cherche à remonter sa natalité et ce, d'une manière unique. Il prend brutalement des mesures d'exception : interdiction de l'avortement et de l'usage de moyens contraceptifs, allocations pour les mères de familles nombreuses... Mécaniquement, la natalité augmente brutalement, mais très temporairement, pour finir par retomber progressivement. La fin du communisme s'accompagne d'une nouvelle baisse de la natalité, jusqu’à seulement 220000 naissances en 2005.

\section{Une mortalité particulièrement élevée}

L'année 2005 enregistre 265000 décès. Le taux brut de mortalité est donc de 12,3 pour mille habitants, un niveau élevé en Europe, puisque le taux moyen de mortalité dans l'Union européenne à 25 est de 9,7 pour mille.

Le phénomène de " surmortalité masculine " s'observe dans tous les groupes d'âges. Chez les 15-64 ans, le taux brut de mortalité de la gent masculine est plus de deux fois plus important que celui des femmes. L'écart entre l'espérance de vie masculine et féminine est supérieur de 7,2 ans en défaveur des hommes. Mais, au début des années 2000, l'espérance de vie des femmes à la naissance atteint 75 ans, le niveau le plus élevé depuis la guerre. Après une tendance en diminution entre 1976 et 1997, l'espérance de vie des hommes est en amélioration depuis 1998, mais à seulement 6768 ans, donc bien inférieure à l'Europe de l'Ouest. Une certaine variation des probabilités de survie des hommes peut être attribuée au stress, au niveau de vie, aux conditions de sécurité sur le lieu de travail, à une mauvaise alimentation, à la pollution ambiante, et à la consommation d'alcool et de tabac.

\section{Une importante émigration comprenant une part importante de minorités}

À compter de 1989, les réformes politiques et sociales déclenchent la levée des restrictions à la libre circulation des personnes d'un pays à l'autre, et des flux migratoires internationaux inconnus jusque-là. Le 
solde migratoire s'affiche constamment négatif, notamment en raison des minorités, qui interviennent sur la composante migratoire, surtout dans le cas de petites régions.

En effet, après 1989, le départ massif de la population d'origine allemande affecte tout particulièrement certaines petites régions du Banat et de Transylvanie. Des communes sont presque totalement délaissées et des villes moyennes de la Transylvanie méridionale très touchées. À l'opposé, les zones où la population tsigane est importante ont une croissance démographique spécifiquement due au comportement très nataliste de ce groupe.

\section{- La minorité Allemande}

Les Allemands de Roumanie descendent des colons (Saxons) venus d'Allemagne au XII siècle pour s'installer en Transylvanie où ils fondèrent des villes comme Brasov. Au lendemain de la fin du rideau de fer en 1989, une grande partie revendiquent le droit du sang afin d'émigrer vers l'Allemagne, laissant derrière eux de véritables no man's land.

\section{- La minorité Hongroise}

La minorité Hongroise (ou Magyare) représente environ deux millions des habitants de la Roumanie (soit près de $9 \%$ ), principalement regroupée en Transylvanie centrale. Une petite partie de cette minorité a émigré vers la Hongrie. Mais, depuis que la Hongrie a intégré l'Union européenne, les territoires roumains proches de la frontière hongroise et majoritairement peuplés par la minorité magyare sont devenus plus dynamiques d'un point de vue économique.
La minorité Roms

Les Roms (appelés également Tsiganes ou Gitans) représentent près d'un million d'habitants. Ils se démarquent du reste des Roumains par leur comportement démographique à natalité élevée et ceci pour des raisons traditionnelles, mais aussi pour des raisons sociales. La pauvreté, associée à la marginalisation sociale de ce groupe ${ }^{8}$, a engendré, face à une politique nataliste très incitative sous Ceausescu, une attitude d'abandon de toute tentative de contrôle de la natalité. De plus, leur situation sociale les conduisent à un mode de vie adapté à la pauvreté et à une natalité élevée. Mais, désormais, un nombre important d'entre eux quitte la Roumanie pour s'installer « à l'Ouest ».

\section{(10}

La croissance démographique roumaine est derrière elle. La politique coercitive de stimulation forcée par Ceausescu ne fut qu'un feu de paille. La Roumanie se dépeuple en raison de son faible taux de natalité et par l'émigration des Roumains, en particulier des Roms, marginalisés. Ainsi, dans les années à venir, la Roumanie sera davantage confrontée au vieillissement généralisé9 (par le haut et par le bas) de sa population. Comme le montre l'analyse démographique de la Roumanie et sachant que l'évolution en Bulgarie est comparable, le sixième élargissement de l'Union européenne concourt à modifier la moyenne des indicateurs du mouvement naturel dans le sens d'une moindre vitalité de l'Union. D'un côté, cette moyenne s'abaisse pour le taux de natalité, l'indice de fécondité ou l'espérance de vie. De l'autre, elle augmente pour le taux de mortalité.
4. Les villes du Sud de la Transylvanie et du Banat, suite à l'émigration massive des populations allemandes, constituaient un cas spécial.

5. La « dépopulation» se définit comme un excédent des décès sur les naissances. Cf. Wackermann, Gabriel (direction), Dictionnaire de Géographie, Paris, Ellipses, 2005.

6. Donc inférieure d'un sixième à celle de la France métropolitaine.

7. Dumont, Gérard-François, Les populations du monde, Paris, Éditions Armand Colin, deuxième édition, 2004.

8. La majorité des Roumains reproche aux Roms d'avoir trop d'enfants par rapport à leurs possibilités de les élever correctement et d'utiliser la natalité élevée comme moyen d'obtenir les prestations sociales.

9. Dumont, Gérard-Francois et alii, Les territoires face au vieillissement en France et en Europe, Paris, Ellipses, 2006. 